Meta

Journal des traducteurs

Translators' Journal

\title{
L'hypertension artérielle et les singularités de son vocabulaire
}

\section{Amal Jammal}

Volume 28, numéro 4, décembre 1983

URI : https://id.erudit.org/iderudit/003244ar

DOI : https://doi.org/10.7202/003244ar

Aller au sommaire du numéro

Éditeur(s)

Les Presses de l'Université de Montréal

ISSN

0026-0452 (imprimé)

1492-1421 (numérique)

Découvrir la revue

Citer cet article

Jammal, A. (1983). L'hypertension artérielle et les singularités de son vocabulaire. Meta, 28(4), 376-391. https://doi.org/10.7202/003244ar d'utilisation que vous pouvez consulter en ligne.

https://apropos.erudit.org/fr/usagers/politique-dutilisation/ 
META, XXVIII, 4

\section{L'HYPERTENSION ARTÉRIELLE ET LES SINGULARITÉS DE SON VOCABULAIRE}

On dit hypertension artérielle et les problèmes sont là, d'emblée. En effet, le préfixe HYPER est médicalement imprécis et le syntagme TENSION ARTÉRIELLE est linguistiquement controversé. Expliquons-nous: si, comme l'expression le dit, l'hypertension artérielle est une tension artérielle supérieure à la normale, on est en droit de se demander ce qu'est la normale. Le fait est que, lorsqu'il s'agit de tension artérielle, la notion de normalité devient très floue. Ce qui est normal pour l'adolescent ne l'est plus pour l'adulte, ce qui est normal le 
soir ne l'est plus le matin, ce qui est normal au repos ne l'est pas après un exercice physique, ce qui est normal pour le Noir ne l'est pas pour le Caucasien. De plus, la tension «normale» differe selon qu'il s'agit d'un homme ou d'une femme, selon que celle-ci est pré- ou post-ménopausée, etc. Bref, les plus conciliants d'entre les disciples d'Esculape reconnaissent que la norme a été fixée de façon arbitraire et qu'elle n'est acceptable qu'assortie d'une foule de «compte tenu de...» (l'âge, la couleur, le sexe, le moment, etc.). Dans ce cas, peut-on encore parler de normalité?

Voilà pour la première difficulté. Examinons maintenant la seconde, soit le syntagme tension artérielle que la plupart des auteurs évitent, lui préférant pression artérielle, plus fidèlement descriptive du phénomène.

Nous savons tous que la circulation sanguine se fait dans un circuit fermé, au centre duquel le cœur agit comme une pompe fonctionnant en trois temps: un premier temps, la diastole (diastole), qui dure environ 0,4 seconde et pendant lequel le cœur est décontracté : les oreillettes et les ventricules se remplissent de sang; un deuxième temps, la systole auriculaire (atrial systole), qui dure environ 0,1 seconde et pendant lequel les oreillettes se contractent et poussent le sang plus activement dans les ventricules et, enfin, un troisième temps, la systole ventriculaire (ventricular systole), qui dure en moyenne 0,3 seconde et pendant lequel les ventricules se contractent et chassent le sang dans les vaisseaux sanguins, le ventricule droit envoyant le sang dans l'artère pulmonaire et le ventricule gauche, avec plus de force, dans l'aorte.

Au moment donc de la systole ventriculaire, lorsque le sang est éjecté avec force dans l'aorte, les parois des artères, surtout de celles qui sont le plus proche du cœur, se distendent et cette distension se propage comme une onde, l'onde sphygmique (pulse wave), permettant la progression du sang dans le réseau vasculaire. Lorsque la contraction ventriculaire prend fin, les parois artérielles étirées reviennent à leur état antérieur et la pression ainsi créée continue de pousser le sang dans le prolongement de plus en plus rétréci des artères, soit les artérioles et les capillaires. Il s'agit donc bien d'une pression artérielle, phénomène physique constituant en quelque sorte la force motrice qui achemine le sang à partir du cœur vers tous les tissus de l'organisme. Obéissant aux lois de la physique, la pression artérielle est, comme toute autre pression exercée par un liquide dans un récipient élastique, fonction de l'élasticité du récipient et du volume du liquide que ce récipient contient. Il est évident qu'ici un troisième élément entre en jeu: la force de contraction de la pompe qui éjecte ce liquide, en l'occurrence le cour.

Voyons maintenant comment, sur le plan linguistique, les spécialistes auteurs de dictionnaires et de manuels médicaux - traitent du problème. Dans le Dictionnaire français de médecine et de biologie, l'entrée descriptive et l'on sait que dans cet ouvrage le choix de l'entrée est significatif - est faite sous pression artérielle avec, à la fin de la définition, l'information suivante: «La tension artérielle (notion biologique) est synonyme de pression artérielle (notion physique) ${ }^{1}$. Plus loin, sous tension artérielle, on peut lire: «1. Dans un

1. MANUILA, A., A.L. MANUILA, M. NICOLE, H. LAMBERT et coll. (1972) : Dictionnaire français de médecine et de biologie en quatre volumes, tome 3, Paris, Masson et Cie, p. 404. 
sens strict, tension des parois artérielles correspondant à la pression sous laquelle circule le sang dans les artères. 2. Par extension, syn. de pression artérielle ${ }^{2}$.

Le Dictionnaire de médecine Flammarion n'honore même pas d'une définition l'expression tension artérielle, mais renvoie laconiquement le lecteur à pression artérielle et, sous cette entrée, à la rubrique «Remarque», souligne ce qui suit :

On assimile presque constamment, dans le langage courant, la pression artérielle (concept physique) à la tension artérielle (concept biologique) qui représente la force élastique exercée par les parois artérielles sur le contenu sanguin pour contrebalancer la pression intramurale. Ces deux expressions sont done devenues synonymes bien qu'elles correspondent à des notions différentes. ${ }^{3}$

En bref, le premier dictionnaire accepte la synonymie, pour ainsi dire, à reculons, alors que le second souligne son impropriété.

Dans un autre ouvrage de référence, le terme tension artérielle est condamné avec force: "C'est par un abus de langage, consacré par l'usage, que les médecins et le public parlent de tension artérielle (et d' «hypertension») alors que, sous ce vocable, ils désignent la pression qui règne à l'intérieur du compartiment artériel ${ }^{4}$.

Quant aux écrits médicaux, on y trouve de tout. Certains auteurs ne parlent que de pression artérielle ${ }^{5,6}, \mathrm{~d}^{\prime}$ autres utilisent indifféremment les deux expressions ${ }^{7}$, d'autres encore font la preuve irréfutable de l'impropriété du terme tension :

Cette double terminologie (tension artérielle et pression artérielle) est critiquable.

$\mathrm{Ce}$ que l'on mesure par la technique du brassard pneumatique est une pression et non une tension artérielle. La Loi de Laplace qui établit la pression et la tension des parois vasculaires selon leur rayon $(P=T / r)$ permet de remarquer que les termes «tension» et «pression» ont des significations bien différentes... il serait préférable d'utiliser le terme pression, qui désigne sans ambiguité le fait hémodynamique que l'on veut caractériser. ${ }^{8}$

Mais alors, une fois tension artérielle condamnée, que doit-on faire des substantifs et adjectifs dérivés de tension, comme hypertension, hypertendu, hypertensif, antihypertenseur? Doit-on leur substituer hyperpression, hyperpressé, hyperpresseur?

Et messieurs les Anglais? Ils parlent bien d'arterial blood pressure et même de blood pressure tout court. Cependant chez eux également la contradiction existe puisqu'ils ont hypertension, hypertensive, antihypertensive.

2. Ibid., p. 924.

3. Dictionnaire de médecine Flammarion, Paris, Flammarion Médecine-Sciences, 1975, p. 602.

4. Encyclopaedia Universalis, vol. 15, Encyclopaedia Universalis France, Paris, 1974, p. 934 à 937.

5. VANDER, A.J., J.H. SHERMAN et D.S. LUCIANO (1977) : Physiologie humaine, Montréal, McGraw Hill Éditeurs, p. 238 à 240.

6. PERLEMUTER, L. et A. CENAC (1977): Dictionnaire pratique de médecine clinique, Paris, Masson, p. 785 .

7. DOUSSET, H. et C. PLARD (1974): Vade-Mecum encyclopédique du médecin praticien, $8^{\mathrm{e}}$ édition, Paris, Maloine S.A. Éditeur, p. 554-555.

8. LUSCKO, M. et L. GUEDON (1973) : «Pression artérielle», Encyclopédie médico-chirurgicale, Paris, fascicule $11301 \mathrm{~A}^{10}$, p. 1 . 
Que peut-on en conclure? Que l'usage est roi et ne se préoccupe guère de logique ou de cohérence.

On ne peut aborder le sujet de l'hypertension artérielle sans parler de l'appareil circulatoire (circulatory system) qui comprend l'appareil cardio-vasculaire (cardiovascular system) et le système lymphatique (lymphatic system). Comme on peut le voir de prime abord, alors que le français utilise les termes appareil et système, pour l'anglais, tout est system. Et pourtant, le terme anglais apparatus existe bel et bien. En réalité, cette disparité naît des différences entre les champs sémantiques anglais et français. Un examen des définitions françaises d'appareil et de système révèle une distinction nette entre les deux termes. En effet, Manuila et coll. donnent du mot appareil la définition suivante : "En anatomie, ensemble des systèmes qui concourent à la même fonction. " ${ }^{9}$ et de système: " 1 . Ensemble de substances, de phénomènes, susceptibles de coexister, d'entrer en réaction, ou de s'influencer réciproquement. 2. Ensemble des organes qui ont une structure analogue. Ex. système osseux. 3. Série complète d'éléments divers constituant une entité biologique ${ }^{10}$.

Si l'on compare la définition d'appareil et la deuxième acception de système, celle qui se rapporte le plus clairement à l'anatomie, aucune ambiguité n'est possible : un système fait partie d'un appareil. Exemple : l'appareil locomoteur (musculoskeletal system) est formé du système osseux (skeletal system), du système musculaire (muscular system) et des articulations (joints).

Qu'en est-il des deux autres acceptions du mot système? La première est bien illustrée par le système rénine-angiotensine (renin-angiotensin system). La rénine est en effet une substance, une enzyme d'origine rénale, qui transforme un polypeptide, l'angiotensinogène (angiotensinogen), en angiotensine I (angiotensin I), laquelle se transforme à son tour en angiotensine II (angiotensin II), une substance hautement vasoconstrictrice, jouant un rôle important dans l'hypertension. On peut constater ici que le premier sens de système s'applique bien au phénomène.

Quant à la troisième acception, assez vague il faut l'avouer, elle peut être illustrée par les différents systèmes de groupes sanguins dont le plus connu est le système Rh.

Du côté anglais, les choses sont beaucoup moins claires: apparatus et system sont considérés comme synonymes avec la préséance accordée à system. Il faut d'ailleurs reconnaître que la distinction entre les deux définitions est beaucoup moins évidente qu'en français. En effet voici ce que nous trouvons dans le Dorland's Illustrated Medical Dictionary pour les deux termes :

apparatus : an arrangement of a number of parts acting together in the performance of some special function; used in anatomical nomenclature to designate a number of structures or organs which act together in serving some particular function ${ }^{11}$.

9. MANUILA, A., A.L. MANUILA, M. NICOLE, H. LAMBERT et coll. (1970) : Dictionnaire français de médecine et de biologie en quatre volumes, tome 1, Paris, Masson et Cie, p. 238.

10. Id. (1972): Dictionnaire français de médecine et de biologie en quatre volumes, tome 3 , Paris, Masson et Cie, p. 889.

11. Dorland's Illustrated Medical Dictionary, 25th edition, Philadelphia, W.B. Saunders Cy., 1974, p. 122. 
system : a set of series of interconnected or interdependent parts or entities (objects, organs or organisms) that function together in a commun purpose or produce results impossible of achievement by one of them acting or operating alone ${ }^{12}$.

En somme, l'élément différenciateur ici est l'interdépendance des parties constituantes, qui semble plus accentuée dans system que dans apparatus. Distinction bien mince en vérité et qui ne résiste guère à un examen serré, car quel est l'«apparatus» dont les composantes ne sont pas interreliées d'une façon ou d'une autre? On est loin de la distinction nette entre le tout et la partie que fait le français.

Il existe cependant, dans le vocabulaire de l'hypertension, un appareil qui fait l'unanimité dans les deux langues, alors même que sa légitimité linguistique semble douteuse puisque certains auteurs ${ }^{13}$ prennent la précaution de le mettre entre guillemets. Il s'agit de l'appareil juxtaglomérulaire (juxtaglomerular apparatus), une formation qui joue un rôle important dans la régulation de la pression artérielle, du fait qu'elle sécrète la rénine dont nous avons parlé plus haut. Voici ce qu'en dit le Dictionnaire de médecine Flammarion :

Formation située au pôle vasculaire du glomérule rénal et comportant: a) les artérioles afférente et efférente; b) le coussin polaire (angl. polar cushion; juxtaglomerular cell mass)... c) la macula densa (angl. macula densa), région cellulaire spécialisée de la paroi du tubule distal, située à l'endroit où ce dernier remonte de la médullaire et passe près du pôle vasculaire du glomérule du même néphron. " ${ }^{14}$

Pourquoi cette formation cellulaire a-t-elle reçu le nom d'appareil ? Est-ce parce que les cellules qui la forment appartiennent à des systèmes régulateurs différents (celles de l'artériole afférente faisant partie du système barosensible alors que celles de la macula densa appartiennent au système chimiosensible)? Ou serait-ce plutôt parce qu'elle comporte des éléments anatomiquement distincts (artériole, tube distal, etc.) ? À notre connaissance, aucun ouvrage n'a encore élucidé la question.

Nous arrivons maintenant à la pièce maîtresse de l'appareil circulatoire : le cœur. Disons-le tout de suite sans fausse modestie : le cœur est un organe francophone! Pour nous en convaincre, il suffit d'ouvrir le Dorland, DICTIONNAIRE UNILINGUE ANGLAIS, à la page 231. On n'y dénombre pas moins de trente bruits français: bruit d'airain (à prononcer: brwe da-ră'), bruit de bois (à prononcer: brwe duh bwah'), bruit de canon (à prononcer: brwe duh kahnaw') et ainsi de suite.

En réalité, bien que la définition du mot anglais bruit soit: «a sound or murmur heard in auscultation, especially an abnormal one», en fait c'est presque exclusivement dans les cas d'anomalie que ce terme est utilisé. Un bruit normal, comme les premier et deuxième bruits du coeur, ceux que provoquent respectivement la fermeture des valvules auriculo-ventriculaires et des valvules aortiques et pulmonaires, sont appelés sound en anglais : first heart sound, second heart sound. Il existe une autre variété de bruit que l'anglais

12. Op. cit., p. $1,533$.

13. Encyclopaedia Universalis, vol. 14, Encyclopaedia Universalis France, Paris, 1974, p. 12.

14. Op. cit., p. 62. 
rend également par sound : ce sont les bruits de Korotkoff, que d'aucuns appellent tons ou phases de Korotkoff ${ }^{15}$ (Korotkoff's sounds), et qui sont perçus lors de la mesure de la pression artérielle par la méthode du sphygmomanomètre : leur apparition indique la valeur systolique et leur disparition la valeur diastolique.

Quant aux autres bruits du cœur, attribuables à des malformations, à des cardiopathies ou à certaines affections pulmonaires, leur terminologie peut facilement désorienter le traducteur. Par exemple, quand le cœur «murmure»-Dieu, que la poésie de ces mots est trompeuse! - il ne le fait pas de la même façon dans les deux langues. En effet, voici comment l'anglais définit murmur: «an auscultatory sound, benign or pathologic, particularly a periodic sound of short duration of cardiac or vascular origin. " ${ }^{16}$ Que le traducteur rende ce terme par murmure et il se sera laissé encore une fois berner par un faux-ami, puisque la plupart du temps, il s'agira d'un souffle, que le Manuila définit comme suit : «Bruit, généralement peu intense, de caractère variable selon l'affection qui en est la cause, mais rappelant plus ou moins le bruit d'une expiration et qui est perçu lors de l'auscultation, en particulier du cour et des poumons. " 17

$\mathrm{Il}$ existe bien un cas ou murmur se traduit par murmure : vesicular murmur, murmure vésiculaire, mais il s'agit alors d'un bruit non pathologique, produit par la vibration normale des alvéoles pulmonaires pendant l'inspiration.

Autre particularité du vocabulaire bilingue du cour: l'imprécision des champs sémantiques de certains termes. Ainsi l'oreillette, c'est-à-dire la cavité du cour où arrive le sang veineux, s'appelle en anglais atrium (du grec atrion et du latin atrium, qui signifient chambre). Oreillette, qui se dit en latin auris, a comme dérivé auriculaire, auriculo-. Atrium, pour sa part, donne comme dérivés atrial, atrio-. Découlent de ce fait deux phénomènes déroutants : en premier lieu, le français utilise parfois l'adjectif atrio-ventriculaire pour désigner une structure nerveuse commune à l'oreillette et au ventricule. Exemple: le noeud atrio-ventriculaire (atrio-ventricular node). Comme on peut le constater, la première composante de l'adjectif français est dérivée du mot anglais atrium. Du côté anglais, le même emprunt étonnant existe puisque l'on peut avoir l'adjectif auriculoventricular, comme dans auriculoventricular groove par exemple. Voilà donc une cavité du cœur dont les dérivés donnent lieu à un chassécroisé linguistique défiant toute logique.

En second lieu, la nomenclature anatomique, tant française qu'anglaise, comporte le vocable auricule (auricle) qui désigne, non pas toute l'oreillette, mais bien une partie de l'oreillette ou, plus précisément, un prolongement diverticulaire de celle-ci; d'où l'ambiguité de l'adjectif auriculaire qui peut se rapporter à la fois à l'oreillette ou à son prolongement, l'auricule.

Il existe une autre anomalie linguistique dans la nomenclature des parties constituantes du cour: la désignation des valves et valvules. On sait que le

15. GARNIER, Marcel et Valéry DELAMARE, Dictionnaire des termes techniques de médecine, $20^{\mathrm{e}}$ édition, Paris, Maloine S.A., p. 703.

16. Dorland's Illustrated Medical Dictionary, 25th edition, Philadelphia, W.B. Saunders Cy., 1974 , p. 989.

17. Op. cit., vol. 3, p. 708 . 
cœur comporte quatre orifices : deux qui permettent la communication entre l'oreillette et le ventricule sous-jacent et deux autres par lesquels, au moment de la systole ventriculaire, le sang est éjecté dans les artères : du ventricule gauche dans l'aorte et du ventricule droit dans l'artère pulmonaire. Ces orifices sont munis de valvules (voir fig. 1) qui sont, comme nous le verrons plus loin, à la fois de faux-amis et une énigme pour le traducteur.

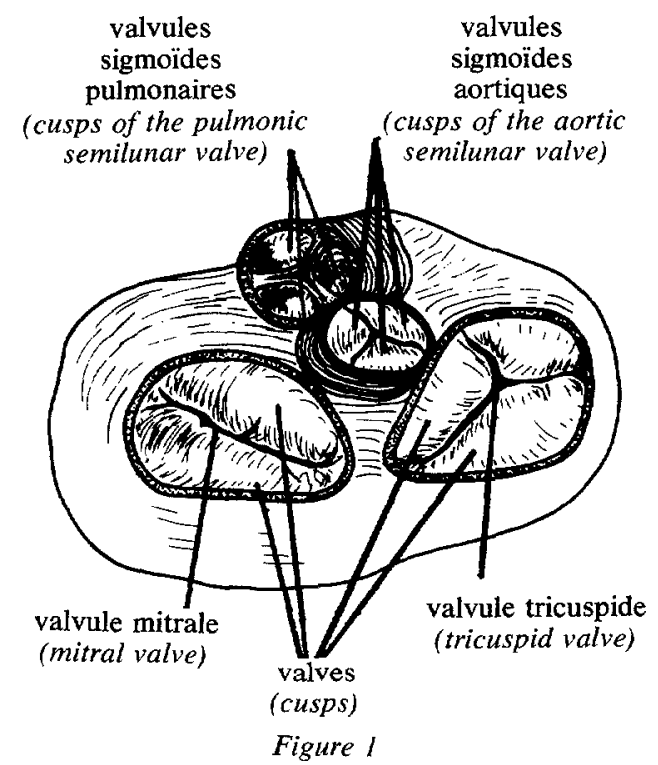

L'orifice auriculo-ventriculaire gauche est muni d'une valvule dite mitrale (mitral valve), ainsi appelée parce que les deux valves (cusps) qui la composent ont vaguement la forme d'une mitre d'évêque. L'orifice auriculo-ventriculaire droit est muni, pour sa part, d'une valvule dite tricuspide (tricuspid valve) parce qu'elle est constituée de trois valves. Jusque-là, mis à part le piège que représente le mot valvule se traduisant en anglais par valve et valve se traduisant par cusp, les correspondances sont sans problème : valvule désigne l'ensemble de la structure et valve chacune des parties qui la composent. Mais les choses commencent à se gâter lorsqu'on en vient aux valvules dont est pourvu chacun des orifices qui font communiquer ventricule et artère. Là, est appelé valvule, non plus l'ensemble de la structure, mais chacun des trois replis semi-lunaires qui la constituent. On a ainsi, au pluriel, les valvules sigmoïdes pulmonaires et les valvules sigmoïdes aortiques. À noter cependant que le Dictionnaire de médecine Flammarion parle de valvule sigmoïde, au singulier, et la définit comme une triple valvule. Le Dorland, plus cohérent, nomme chacune de ces structures semilunar valve et la définit comme suit: «a valve having semilunar cusps, i.e., the aortic valve and the pulmonary valve;" mais ajoute, pour ne pas être en reste sur la confusion de la terminologie française: «The term is sometimes used to designate the semilunar cusps composing these valves.» ${ }^{18}$ Pour semilunar

18. Op. cit., p. 1690. 
cusp, il donne: «any of the semilunar segments of the aortic valve... or the pulmonary valve». Et, là encore pour ne pas déroger à la règle de l'ambiguiité, ajoute: "called also valvula semilunaris ${ }^{19}$.

Que saint Jérôme nous vienne en aide!

En suivant le circuit de la révolution cardiaque, on arrive aux vaisseaux sanguins. Leur terminologie présente également quelques curiosités, dont certaines ont trait à leur désignation et d'autres, à la manière dont les anatomistes les ont divisés.

Que l'on nous pardonne de rappeler tout d'abord certaines notions de base: On appelle sang artériel le sang chargé d'oxygène, et sang veineux, le sang chargé de gaz carbonique. Le sang est donc qualifié ici d'après le type de vaisseaux dans lequel il circule - artère ou veine - et cette qualification est directement reliée à sa composition chimique. On s'attendrait donc, en toute logique, à l'équation inverse suivante : une artère est un vaisseau qui transporte du sang artériel, c'est-à-dire chargé d'oxygène, et une veine est un vaisseau qui transporte du sang veineux, c'est-à-dire chargé de gaz carbonique. Or, il n'en est rien, puisque, lorsqu'on en vient à la désignation des vaisseaux sanguins, celle-ci n'est plus fonction de la composition chimique du contenu du vaisseau, mais plutôt de la DIRECTION de ce contenu. Autrement dit, on appelle artère tout vaisseau qui transporte du sang allant du cœur vers le reste de l'organisme, et veine tout vaisseau qui ramène le sang venant de l'organisme vers le cœur; et cela, indépendamment de la qualité du sang que le vaisseau contient. On peut donc avoir l'artère pulmonaire qui transporte du sang veineux et les veines pulmonaires qui transportent du sang artériel!

On rencontre par ailleurs une autre anomalie, de correspondance cette fois, entre les nomenclatures française et anglaise des artères. Elle naît dans certains cas d'un vide terminologique, et, dans d'autres, d'une divergence dans le «découpage» anatomique de certains segments de l'arbre artériel.

D'abord, la crosse aortique. La nomenclature française divise ce segment en deux sous-segments (voir fig. 2): l'aorte ascendante, qui va de l'orifice aortique du ventricule gauche jusqu'au début de la partie horizontale de la crosse, et l'arc de l'aorte, segment horizontal de la crosse qui se continue par l'aorte descendante.

La nomenclature anglaise divise bien cette structure anatomique en ascending aorta et arch of the aorta, mais elle n'a pas d'équivalent pour l'ensemble, c'est-à-dire pour la crosse aortique. À cause de cette lacune, une certaine confusion règne, que le Dictionnaire de médecine Flammarion augmente à plaisir. En effet, alors que le Dorland définit comme suit le terme anglais arch of the aorta: «the continuation of the ascending aorta... ${ }^{20}$ et fait ainsi la preuve d'une correspondance parfaite entre arc de l'aorte et arch of the aorta, on peut lire dans le Flammarion: "Crosse de l'aorte (angl. arch of aorta). Portion initiale de l'aorte... formée de deux parties : l'une ascendante, l'autre horizontale... »21

19. Ibid., p. 391 .

20. Op. cit., p. 129.

21. Op. cit., p. 58. 


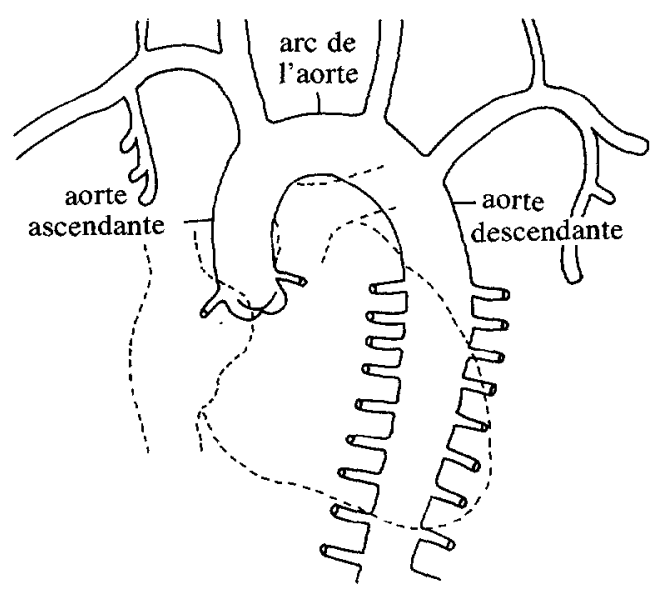

Figure 2 - La crosse aortique

Plus loin, le même dictionnaire donne, comme équivalent anglais de crosse aortique droite ou dextroposition de l'aorte*, right aortic arch, et comme équivalent de syndrome de la crosse aortique, aortic arch syndrome.

Un peu plus bas, dans l'arbre artériel, on retrouve une autre divergence de nomenclature entre les deux langues, divergence qui peut faire perdre un temps précieux au traducteur. En effet, si celui-ci tente de trouver un équivalent anglais au terme tronc tibio-péronier il en sera pour sa peine. L'expression n'existe pas. Là, le phénomène est imputable à une différence dans la division anatomique. Le français appelle tronc tibio-péronier la portion d'artère qui prolonge l'artère poplitée et qui, d'après la nomenclature française, se divise après un court trajet en artère tibiale postérieure et artère péronière; d'où l'appellation de tronc pour la partie commune qui précède la division (pour plus de détails au sujet de l'arbre artériel, voir l'ouvrage intitulé l'Hypertension artérielle ${ }^{22}$.

Les anatomistes anglais, par contre, divisent autrement cette partie du système artériel. Ils considèrent que l'artère poplitée se prolonge par l'artère tibiale postérieure et que celle-ci a, comme artère collatérale - c'est-à-dire qui s'en détache - l'artère péronière. Donc, pas de tronc commun et pas d'équivalent anglais à tronc tibio-péronier (voir fig. 3).

Nous passons maintenant à la terminologie de la circulation sanguine. Comme chacun le sait, on distingue la circulation pulmonaire ou petite circulation (pulmonary circulation) qui conduit le sang pour le purifier vers les poumons et l'en ramène chargé d'oxygène vers le cœur, et la grande circulation (systemic circulation) qui irrigue toutes les parties du corps sans exception.

* Nous mettons en doute par ailleurs la synonymie de ces deux termes qui, à notre avis, désignent deux malformations différentes.

22. JAMMAL, Amal et F. Pierre DUSSEAULT, l'Hypertension artérielle, collection Initiation aux langues de spécialités, Montréal, Sodilis, 1983, p. 9 à 12. 


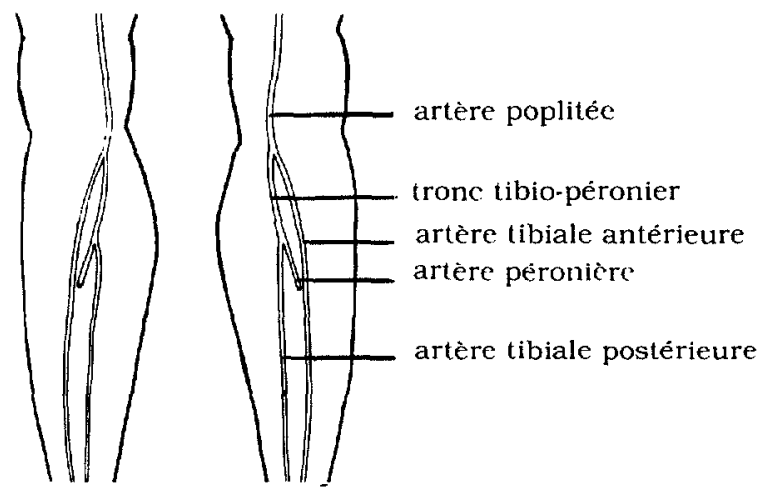

Figure 3-Découpages anatomiques français et anglais des artères tibiale et péronière.

Rendre donc, comme le font certains, l'expression anglaise systemic circulation par circulation systémique, c'est non seulement commettre un anglicisme mais, à la limite, aller vers un contresens. En effet, si systémique désigne ce qui se rapporte à un système, il ne peut que s'opposer à général. Une circulation systémique désignerait en réalité une circulation qui irrigue non pas le corps tout entier, mais un système en particulier, comme le système porte par exemple. À ce sujet, la lecture de la définition du mot systémique donnée par le Flammarion laisse rêveur: «Systémique adj.: 1.(angl. systemic). Qui se rapporte à un système ou à des éléments appartenant à un même système. 2. (Cardiol.) (pas d'équivalent anglais) [sic]. Qui se rapporte à la grande circulation ou aux cavités cardiaques gauches. ${ }^{23}$

Non seulement on donne droit de cité à un anglicisme patent, mais on affirme de plus que la source de cet anglicisme est inexistante!

Nous arrivons enfin à la terminologie de l'hypertension proprement dite. Là, les curiosités linguistiques abondent. L'hypertension artérielle peut être qualifiée de légère (mild), de modérée (moderate) ou de grave (severe). Elle peut être aussi qualifiée de bénigne (benign) ou de maligne (malignant). Est-ce à dire qu'on peut automatiquement établir, comme on serait tenté de le faire, une certaine synonymie entre légère et bénigne d'une part, et grave et maligne d'autre part; utiliser, dans chaque groupe, l'une ou l'autre expression indifféremment? Nenni! Les qualificatifs légère, modérée ou grave indiquent chacun une fourchette de chiffres tensionnels, établie arbitrairement et pas toujours de façon uniforme :

$\begin{array}{ll}\text { Hypertension légère } & \begin{array}{l}\text { de } 90 \text { à } 100 \mathrm{~mm} \text { de mercure } \\ \text { Hypertension modérée }\end{array} \\ \text { de } 100 \text { à } 120 \mathrm{~mm} \text { de mercure } \\ \text { Hypertension grave } & >120 \mathrm{~mm} \text { de mercure }\end{array}$

Les qualificatifs bénigne et maligne ont trait par contre au degré de gravité des manifestations de l'hypertension et à son évolution, Hypertension bénigne : absence de manifestations graves, évolution lente; hypertension maligne : manifes-

23. Op. cit., p. 703.

24. MANN, James L. (1974) : "Antihypertensive therapy today " Drug Merchandising, février. 
tations graves, comprenant artériolite nécrosante, tension diastolique élevée, dilatation du ventricule gauche, rétinopathie, etc., et évolution rapide vers la mort.

Qu'elle soit modérée ou grave, maligne ou bénigne, essentielle - c'est-àdire sans étiologie décelable - ou secondaire - c'est-à-dire d'origine rénale, vasculaire, gravidique, surrénalienne ou autre - l'hypertension requiert des soins. Mais des soins pour... quoi au juste? La question est évidemment linguistique. L'anglais n'est pas en peine, il dispose du verbe omnibus to control et répondra donc to control hypertension. Comment rendre en français cette expression, si l'on veut éviter l'anathème que les puristes jettent sur l'usage du verbe contrôler, entendu autrement que dans le sens de vérifier, inspecter? On peut toujours dire abaisser les chiffres tensionnels, mais cette solution n'est pas sans faille : d'abord, abaisser est dépourvu de l'aspect duratif contenu dans to control, ensuite, on n'y perçoit pas l'aspect implicite de retour à la normale suggéré par le terme anglais. Mais alors? Ramener et maintenir à un niveau normal la tension artérielle? C'est trop long et se substantive très mal. Juguler l'hypertension? Le terme va bien pour une maladie mais pour l'hypertension qui, rappelons-le n'est pas une maladie, il semble un peu fort et par trop définitif. Il reste maîtriser l'hypertension qui a la faveur de la gente traductrice mais qui n'est pas sans défaut puisque, parmi les acceptions du Robert, la seule que l'on pourrait appliquer ici, à condition que l'on ne soit pas trop exigeant, est la suivante : «Maîtriser les forces de la nature... Maîtriser un incendie, un fléau, l'arrêter».

On pourrait se demander pourquoi on n'emboiterait pas le pas au même Robert qui, dans sa dernière édition, n'accuse même plus contrôle, pris dans ce sens, d'être un anglicisme, et traduire to control hypertension par contrôler l'hypertension? La tentation est grande et le seul obstacle que l'on pourrait y voir vient du fait que le traitement de l'hypertension exige du médecin qu'il contrôle (dans le sens de vérifie cette fois) régulièrement les chiffres tensionnels de son patient. D'où une ambiguïté possible, d'où peut-être la bonne fortune de l'expression maitriser l'hypertension.

Un autre problème terminologique, relié au traitement de l'hypertension, a trait au bon vouloir de l'hypertendu. Tous les médecins le reconnaissent: pour que le traitement ait des chances de réussir, il faut que le principal intéressé se présente fidèlement aux rendez-vous, prenne régulièrement les médicaments prescrits et suive les recommandations du médecin quant au régime alimentaire, à l'exercice physique, à l'usage du tabac et de l'alcool, etc. Autrement dit, il est indispensable que le médecin obtienne ce que les anglophones appellent the patient's compliance. Gare aux faux-amis! Il n'est nullement question ici de compliance en français: ce terme, qui est par ailleurs introuvable dans le Robert, appartenant exclusivement à la langue de la physique et, par extension, à celle de la médecine signifie en effet :

«Grandeur analogue à une impédance, qui mesure le rapport de l'accroissement de volume $(\Delta \mathrm{V}$ en $\mathrm{ml})$ fonction d'une variation de pression $(\Delta \mathrm{P})$ à cette variation $\left(\Delta \mathrm{P}\right.$ en $\mathrm{cm}$ d'eau) ${ }^{2 s}$

$\overline{25 . ~ D i c t i o n n a i r e ~ d e ~ m e ́ d e c i n e ~ F l a m m a r i o n, ~ p . ~} 178$. 
Limpide... mais de toute évidence ne s'appliquant pas au bon vouloir du patient. Si nous voulons retomber dans la langue courante et chercher dans le Harrap's l'équivalent de compliance, nous trouvons :

«Compliance : 1. Acquiescement (with, à). 2. Péj. (Base) compliance: soumission (abjecte)...»

De toute évidence, l'acquiescement ici est insuffisant parce que pas assez actif et la soumission, même quand elle n'est pas abjecte, a un relent d'aliénation tout à fait inacceptable.

On peut certes, en étendant un peu le champ sémantique recouvert par compliance et en y ajoutant un trait plus actif, utiliser collaboration ou coopération. Tout va bien jusqu'à ce qu'on se trouve face, non plus au substantif, mais au verbe : "The patient must comply with the therapeutic regimen set by his doctor». Peut-on traduire par : «Le patient doit acquiescer - ou collaborer au régime thérapeutique établi par son médecin»? Il peut acquiescer et ne pas le suivre. Quant à collaborer au régime, l'expression est en nette contradiction avec établi par son médecin. En fait, ce qu'on demande au patient c'est de suivre fidèlement le régime thérapeutique qui lui est prescrit. Donc, selon que l'expression anglaise prenne la forme substantive ou verbale, on peut ménager ou non la susceptibilité du patient, puisque, dans le premier cas, on parle de sa collaboration et, dans le deuxième, de sa ... Au fait comment peut-on substantiver suivre fidèlement? Stricte observance? C'est un co-occurrent de lois et de rites. Conformité? Le terme est accompagné dans le Robert de la mention « $\mathrm{Vx} »$.

Et quand le patient ne collabore pas? Lorsqu'il se lasse du traitement et «laisse tomber»? L'anglais utilise l'expression to drop out (from the treatment) qu'il est facile de traduire par abandonner le traitement. Mais arrive l'expression a dropout pour désigner le patient qui a abandonné son traitement et les problèmes surgissent. Le Service linguistique de Radio-Canada nous propose décrocheur et défectionnaire, pour désigner : «une personne qui cesse de s'intéresser à quelque chose, par découragement, lassitude, mécontentement ou tactique. ${ }^{26}$

Le premier qui est accompagné de la mention «familier» passerait à la rigueur en publicité mais s'insèrerait mal dans un article sérieux. Dans ce dernier cas, il faudrait sans doute lui préférer défectionnaire.

En résumé, selon le contexte, a dropout se traduirait par un décrocheur ou un défectionnaire, mais l'acte lui-même, to drop out, par abandonner le traitement.

Examinons enfin les particularités terminologiques propres à l'exploration diagnostique et à l'arsenal thérapeutique antihypertensif.

Parmi les multiples examens effectués par le médecin pour le dépistage de l'hypertension figure l'examen du fond de l'oeil ou du fond d'oeil (optic fundus). L'expression fond d'oeil est souvent utilisée, malgré sa construction insolite qui rappelle étrangement fond de tiroir ou fond de marmite. Le cas est assez exceptionnel; partout ailleurs, le mot fond est suivi d'un nom déterminé :

26. Fiche de Radio-Canada $n^{\circ} 334,1974$ 
fond de l'estomac (fundus ventriculi), fond de la vésicule biliaire (fundus of gall bladder), fond de l'utérus (fundus uteri), etc. Seul fond d'oeil déroge à la règle.

Dans ce fond de l'œil donc, le médecin recherche, entre autres, un signe qui se dit en anglais nicking et pour lequel il n'existe pas de vocable français aussi réduit. Le traducteur se trouve contraint d'utiliser, comme équivalent, la définition même du terme, soit rétrécissement localisé des vaisseaux de la rétine dans l'hypertension artérielle ${ }^{27}$.

Une fois l'hypertension diagnostiquée, le médecin choisit le ou les médicaments qui lui semblent les plus efficaces. Parmi ceux-ci, il en est qui, sur le plan terminologique, ont des synonymes admis par l'usage mais sémantiquement contestables. Nous avons en premier les bêta-bloquants, dits encore bêtabloqueurs ou agents bêta adrénolytiques ou bloqueurs des récepteurs bêtaadrénergiques. Du côté anglais, on a le choix entre beta blockers, beta adrenergic receptor blocking agents, beta adrenergic blocking agents, beta blocking agents. En gros, il s'agit de substances capables de bloquer des récepteurs adrénergiques, c'est-à-dire des zones dont la structure moléculaire réagit habituellement à l'action de certains médiateurs chimiques, dont l'adrénaline et la noradrénaline. On divise ces récepteurs en récepteurs alpha et récepteurs bêta. La stimulation de ces derniers provoque, entre autres, l'accélération du rythme cardiaque. Certaines substances, en inhibant cette stimulation, peuvent empêcher l'élévation de la pression artérielle. L'appellation la plus correcte, pour ces substances, serait donc agents bloqueurs des récepteurs bêta-adrénergiques, appellation qui serait parfaitement descriptive mais un peu longue. D'où la contraction bêta-bloquant. Il reste à déterminer si cette contraction est justifiée. Elle l'aurait été pleinement si le préfixe bêta était exclusivement accolé à récepteur adrénergique. Or, il n'en est rien puisque l'on a bêta-lactamase, bêtaglobuline pour ne citer que ceux-là. Donc bêta-bloquant pèche par manque de précision. Ceci dit et l'usage étant tout-puissant, faut-il dire bêta-bloquant ou bêta-bloqueur? Pourquoi la préférence accordée à bêta-bloquant et manifestée tant par son choix comme mot-vedette ${ }^{28}$ que par la mise entre parenthèses de bêta-bloqueur ${ }^{29}$ ?

Dans les deux cas pourtant les suffixes dérivationnels eur et ant signifient «qui fait l'action». On a, sur les mêmes modèles, convulsivant - qui provoque des convulsions - et inhibiteur - qui provoque l'inhibition. Serait-ce que eur comporte un aspect plus actif? Si tel est le cas, dans bêta-bloquant, le suffixe impliquerait que bêta est l'objet du blocage, alors que dans bêta-bloqueur, il laisserait entendre que bêta est l'agent du blocage, ce qui serait totalement faux. D'où peut-être la faveur que connaît bêta-bloquant.

Avec l'avancement de la recherche, on tente de traiter l'hypertension, non plus à l'aveuglette, mais de façon sélective, c'est-à-dire en agissant sur la structure organique que l'on soupçonne à l'origine du trouble. C'est ainsi que le

27. GLADSTONE, E.J., Dictionnaire anglais-français des sciences médicales et paramédicales, St-Hyacinthe, Edisem Inc: et Paris, Maloine S.A., 1978, p. 664.

28. Dictionnaire de médecine Flammarion, p. 100.

29. MANUILA, A., L. MANUILA et J.C. SOURNIA (1981): Dictionnaire français de médecine et de biologie, Supplément, Paris, Masson, p. 31. 
médecin choisit l'un ou l'autre de ces bêta-bloquants selon leur mode d'action ou selon les effets qu'il recherche : cela peut-être la cardio-sélectivité s'il veut agir sélectivement sur les récepteurs cardiaques, ou un effet dépresseur sur le myocarde que l'on obtient par le biais d'une action sur les échanges électrolytiques qui se font à travers la membrane cellulaire. Pour qualifier ce deuxième effet, que les dictionnaires n'ont pas encore nommé, l'usage, tant français qu'anglais, est hésitant. C'est ainsi que l'on peut lire en anglais membrane stabilizing effect mais aussi membrane effect, et en français effet stabilisateur des membranes, mais aussi effet stabilisant de membrane, dont la structure est source d'ambiguité, et effet de membrane, aussi elliptique qu'abscons.

Enfin, les derniers venus dans la pharmacothérapie antihypertensive: les substances qui agissent sur les canaux d'entrée du calcium et, par ricochet, sur la régulation du tonus des muscles lisses des vaisseaux sanguins. Là également, la terminologie n'est pas fixée; elle varie d'un auteur à l'autre. On rencontre antagonistes du calcium, appellation qui serait impropre puisqu'en fait ces substances n'antagonisent pas le calcium, mais en bloquent plutôt l'entrée, bloqueurs des canaux du calcium ou des canaux calciques, bloqueurs d'entrée du calcium. Voilà une synonymie qui reste à défricher.

Nous n'avons pas la prétention d'avoir fait le tour des particularités du vocabulaire de l'hypertension. Celles-ci sont appelées à se multiplier, d'abord parce que la recherche sur le sujet progresse très rapidement, ensuite parce que les chercheurs ne s'embarrassent guère de scrupules linguistiques, enfin, parce que la terminologie ne peut en quelque sorte qu'agir après le fait et éprouve parfois des difficultés considérables à déloger des termes incorrects mais que l'usage a eu le temps d'introniser.

A. JAMMAL

\section{BIBLIOGRAPHIE}

Atlas d'anatomie élémentaire (1979), Paris, Librairie Maloine S.A. èditeur et Saint-Hyacinthe, Edisem, 32 planches.

BEST \& TAYLOR (1979): Physiological Basis of Medical Practice, edited by John R. Brobeck, 10 th edition, Baltimore, The William \& Wilkins Company, $1178 \mathrm{p}$.

BEVELANDER, Cerrit (1973): Essentials of Histology, $6^{\text {th }}$ edition, St-Louis, Missouri, The C.V. Mosby Company, 1970, traduit par André J. Collet sous le titre de Élements d'histologie, Paris, Maloine S.A. éditeur, 335 p.

BLOOM, William \& Don FAWCETT (1975): A Textbook of Histology, Philadelphia, W.B. Saunders Cy., 1033 p.

CARATINI, Roger (1974): Atlas d'anatomie humaine, Encyclopédie thématique universelle Bordas, Paris-Bruxelles-Montréal, Bordas-Éditeur, 124 planches.

Dictionnaire de médecine Flammarion (1975), Paris, Flammarion Médecine-Sciences, 874 p.

Dorland's Illustrated Medical Dictionary (1974), $25^{\text {th }}$ edition, Philadelphia, W.B. Saunders Cy., $1748 \mathrm{p}$.

DOUSSET, H. et C. PLARD (1974): Vade-Mecum encyclopédique du médecin praticien, $8^{\mathrm{e}}$ édition, Paris, Maloine S.A., Editeur, 'Les fiches terminologiques du Service linguistique' de Radio Canada.

Encyclopaedia Universalis (1974) : vol. 14, Encyclopaedia Universalis France, Paris, $1069 \mathrm{p}$. Encyclopaedia Universalis (1974): vol. 14, Encyclopaedia Universalis France, Paris, 1104 p. Encyclopaedia Universalis (1974): vol. 15, Encyclopaedia Universalis France, Paris, 1105 p. Encyclopédie médico-chirurgicale, Cœur. Vaisseaux., Paris, fascicules 1.1313 A10, 11001 A10, 11001 D10, 11001 E10 et 11001 F10. 
GARNIER, Marcel et Valéry DELAMARE (1978): Dictionnaire des termes techniques de médecine, 20 édition, Paris, Maloine S.A., 1340 p.

GLADSTONE, W.J. (1978): Dictionnaire anglais-français des sciences médicales et paramédicale s/English-French Dictionary of Medical and Para-medical Sciences, St-Hyacinthe, Edisem Inc. et Paris, Maloine S.A., 1535 p.

GUNTZ, M. (1975) : Nomenclature anatomique illustrée, Paris, Masson et Cie, Éditeurs, $494 \mathrm{p}$.

GUYTON Arthur, (1981): Textbook of Medical Physiology, $6^{\text {th }}$ edition, PhiladelphiaLondon-Toronto, W.B. Saunders Cy., 1074 p.

Harrap's Shorter French and English Dictionary (1967): Toronto \& Vancouver, Clarke, Irwin \& Company Ltd.

HARRISON, T.R. (1982): Principes de médecine interne, vol. 2, 3e édition française, traduction de la 9 e édition américaine, Chevilly-Larue (France), Flammarion Médecinesciences, $2282 \mathrm{p}$.

JAMMAL, Amal et F. Pierre DUSSEAULT (1983): l'Hypertension artérielle, collection Initiation aux langues de spécialités, Montréal, Sodilis, $89 \mathrm{p}$.

LACOMBE, M. (1974) : Précis d'anatomie et de physiologie humaines, tome 1, 12e édition, Paris, Éditions Lamarre-Poinat, $190 \mathrm{p}$.

LACOMBE, M. (1974): Précis d'anatomie et de physiologie humaines, tome II, $12^{\mathrm{e}}$ édition, Paris, Éditions Lamarre-Poinat, 145 p.

LUSCKO, M. et L. GUEDON (1973): "Pression artérielle», Encyclopédie médicochirurgicale, Paris, fascicule $11301 \mathrm{~A}^{10}$.

MANN, James L. (1974) : "Antihypertensive therapy today in Drug Merchandising, février.

MANUILA, A., A.L. MANUILA, M. NICOLE, H. LAMBERT et coll. (1970) : Dictionnaire français de médecine et de biologie en quatre volumes, tome 1, Paris, Masson et Cie, $865 \mathrm{p}$.

MANUILA, A., A.L. MANUILA, M. NICOLE, H. LAMBERT et coll. (1971) : Dictionnaire français de médecine et de biologie en quatre volumes, tome II, Paris, Masson et Cie, $923 \mathrm{p}$.

MANUILA, A., A.L. MANUILA, M. NICOLE, H. LAMBERT et coll. (1972) : Dictionnaire français de médecine et de biologie en quatre volumes, tome III, Paris, Masson et Cie, 1193 p.

MANUILA, A., A.L. MANUILA et J.C. SOURNIA (1981) : Dictionnaire français de médecine et de biologie. Supplément, Paris, Masson, $192 \mathrm{p}$.

MAURICE, P., F. FERNANDEZ et P. OUBRAK (1979): Cardiologie-physiopathologie clinique, $2^{\mathrm{e}}$ édition, Paris, Éditions J.B. Baillière, $391 \mathrm{p}$.

PERLEMUTER, L. et A. CENAC (1977) : Dictionnaire pratique de médecine clinique, Paris, Masson.

ROBERT, Paul (1981): Le Petit Robert, Dictionnaire alphabétique et analogique de la langue française, Paris, Le Robert, $2171 \mathrm{p}$.

ROUVIĖRE, H. (1970): Anatomie humaine, $11^{\mathrm{e}}$ édition, Paris, Masson et Cie Éditeurs, $616 \mathrm{p}$.

The Ciba Collection of Medical Illustrations (1978): vol. 5 - The Heart - prepared by Frank H. Netter, edited by Frederick F. Yankman, U.S.A., Ciba Pharmaceutical Company, Division of Ciba-Geigy Corporation, 295 p.

The Merck Manual of Diagnosis and Therapy, (1977): 13th edition, Robert Berkow M.D. Editor, Eahway, N.J., published by Merck Sharp \& Dohme Research Laboratories, Division of Merck \& Co., $2165 \mathrm{p}$.

VANDER, A.J., J.H. SHERMAN et D. LUCIANO (1977) : Physiologie humaine, Montréal, McGraw Hill Éditeurs, $608 \mathrm{p}$. 